\title{
Investigation of Factors Affecting Body Temperature Changes During Routine Clinical Head Magnetic Resonance Imaging
}

\author{
Myeong Seong Kim,"* \\ ${ }^{1}$ Department of Radiology, The Korean National Cancer Center, Goyang-si, Republic of Korea \\ "Corresponding author: Myeong Seong Kim, Department of Radiology, The Korean National Cancer Center, Goyang-si, Republic of Korea. Tel: +82-319201195, Fax: +82-319201171, \\ E-mail: fame6656@ncc.re.kr
}

Received 2015 October 23; Revised 2016 March 17; Accepted 2016 May 02.

\begin{abstract}
Background: Pulsed radiofrequency (RF) magnetic fields, required to produce magnetic resonance imaging (MRI) signals from tissue during the MRI procedure have been shown to heat tissues.

Objectives: To investigate the relationship between body temperature rise and the RF power deposited during routine clinical MRI procedures, and to determine the correlation between this effect and the body's physiological response.

Patients and Methods: We investigated 69 patients from the Korean national cancer center to identify the main factors that contribute to an increase in body temperature (external factors and the body's response) during a clinical brain MRI. A routine protocol sequence of MRI scans ( $1.5 \mathrm{~T}$ and $3.0 \mathrm{~T}$ ) was performed. The patient's tympanic temperature was recorded before and immediately after the MRI procedure and compared with changes in variables related to the body's physiological response to heat.

Results: Our investigation of the physiological response to RF heating indicated a link between increasing age and body temperature. A higher increase in body temperature was observed in older patients after a 3.0-T MRI $(r=0.07, P=0.29$ for $1.5-\mathrm{T}$ MRI; $r=0.45, P$ $=0.002$ for 3.0-T MRI). The relationship between age and body heat was related to the heart rate (HR) and changes in HR during the MRI procedure; a higher RF power combined with a reduction in HR resulted in an increase in body temperature.

Conclusion: A higher magnetic field strength and a decrease in the HR resulted in an increase in body temperature during the MRI procedure.
\end{abstract}

Keywords: Magnetic Resonance Imaging, Body Temperature, Heart Rate

\section{Background}

Magnetic resonance imaging (MRI) is a commonly used method of imaging soft tissue contrast (1). Unlike X-ray-based medical diagnostic techniques such as computed tomography, MRI does not use ionizing radiation. MRI scanners have to date been considered safer (ionizing radiation-free) than other radiology diagnostic modalities. However, the frequency of MRI procedures has increased dramatically, with over 27.5 million studies in the United States (US) in 2007 (2). Moreover, clinicians desire higher signal-to-noise (SNR) ratios for high-resolution MRI (3-5). To meet these needs, the magnetic field strength used for MRI has increased gradually (6).

Nevertheless, the use of MRI presents potential safety hazards, including strong static magnetic fields, gradient magnetic fields, and pulsed radio-frequency (RF) fields (7, 8). The US Food and Drug Administration (US-FDA, Washington, DC) (9-11) and various studies report that static and gradient magnetic fields have no adverse effects on mammals up to 8 Tesla $(\mathrm{T})(10,12,13)$.
However, pulsed RF magnetic fields, required to produce MR signals from tissue during the MRI procedure have been shown to heat tissues (14-17). The major hazards associated with MRI, such as exhaustion and heat stroke, are attributed to the thermal effects of $\operatorname{RF}(18,19)$. Additionally, clinical 3.0-T MRI scans may have genotoxic effects on cultured human lymphocytes (20).

MR imagery is acquired by reconstructing the precession of hydrogen nuclei magnetic moments from the RF signal. This precession of magnetic moments depends on the gyromagnetic ratio. For hydrogen protons, the gyromagnetic ratio is equal to $42.6 \mathrm{MHz} \mathrm{T}^{-1}$; thus, the frequencies of the pulsed RF field are 63.6 and $127.6 \mathrm{MHz}$ for 1.5 and 3.0-T scans, respectively.

The RF radiation absorption rate during an MRI is typically described by the specific absorption rate (SAR), expressed in watts per kilogram $\left(\mathrm{W} \mathrm{kg}^{-1}\right)$. It has been also reported that the SAR is four-fold greater with 3.0-T MRI, compared with that of a 1.5-T scan, using the same pulse sequence $(4,21,22)$. The US FDA restricts the amount of heat that can be induced in human tissue $(8,12,23)$. Unac- 
ceptable levels are reached more rapidly with 3.0-T systems than with 1.5 -T systems $(12,24)$. Thus, the potential for thermal injury from excessive RF power deposition exists $(7,24$, 25).

Other physiological responses related to body temperature rise during an MRI scan have been observed in patients with impaired cerebral perfusion, such as stroke, tumors, or arteriovenous malformations $(12,26)$. The potential increase in body temperature is also affected by patient age, heart rate (HR), body size and type, as well as the loss of body heat through metabolic processes (radiation, convection, and evaporation of sweat and ventilation, and humidity response to heat stress) $(26,27)$. Additionally, the HR value decreases as the patient's age increases (28-31). The response of the body to heat stress is influenced by multiple physiological and environmental factors $(12,13,32,33)$.

The increase in body temperature related to RFabsorption is well known. However, most studies used animal models in their evaluation of heat elevation by RF absorption. Few studies have been published regarding human physiological changes resulting from RF exposure during clinical use of MRI (1.5- or 3.0-T MRI) or routine sequence protocol (commonly used clinical sequence parameter). Previous studies have indicated that the body temperature increase is dependent on the SAR value of the MRI system $(12,15,24,34)$. We hypothesize that the body temperature increase is dependent on the RF power deposited by the MRI system and the patient's physiological characteristics $(12,35)$.

\section{Objectives}

The main objective of this study was to examine the vital signs of the patient, such as HR, oxygen saturation, age, sex, and body mass index (BMI) to find the association between these factors and the body's physiological response during 1.5- or 3.0-T head MRI scans $(12,25)$. Therefore, we attempted to resolve the difference in the physiological response between 1.5- and 3.0-T scans, as well as determine which physiological response has a greater effect on increased body temperature when applied to a routine head MRI protocol for general use in the clinic.

\section{Patients and Methods}

\subsection{Patients}

Sixty-nine subjects (38 with imagery interpretation results confirming brain tumors and 31 normal brain patients) ranging from 10 to 77 years of age were selected at random from patients who visited the Korean national cancer center (NCC), Goyang-si, Republic of Korea. Table 1 shows a detailed description of patient classification. The patients were enrolled between December 2009 and March 2010 at the NCC. We received consent from the patients for participation in the study under clinical-MRI routine brain protocols and had the approval of the NCC Institutional Review Board (IRB).

Table 1. Patient Characteristics ${ }^{\mathrm{a}}$

\begin{tabular}{|lc|}
\hline Characteristics & Results $(\mathbf{n}=\mathbf{6 9})$ \\
\hline Age $^{\mathbf{b}}$ & $54.72 \pm 12(16-77)$ \\
\hline Weight $^{\mathbf{b}}$ & $64.83 \pm 8.3(44-77)$ \\
BMI $^{\mathbf{b}}$ & $22.9 \pm 2.5(18-28.7)$ \\
\hline Gender & \\
\hline \multicolumn{1}{|c|}{ Male } & 33 \\
\hline Female & 36 \\
\hline Field Strength & \\
\hline $1.5 \mathrm{~T}(16 \mathrm{~kW})$ & 21 \\
\hline $3.0 \mathrm{~T}(25 \mathrm{~kW})$ & 36 \\
\hline $3.0 \mathrm{~T}(35 \mathrm{~kW})$ & 12 \\
\hline Brain Tumor & 38 \\
\hline Yes & 31 \\
\hline No & \\
\hline
\end{tabular}

Abbreviation: BMI, body mass index

${ }^{\mathrm{a}}$ Status of brain tumor: confirmed by magnetic resonance imaging (MRI) results

${ }^{\mathrm{b}}$ Data are expressed as means $\pm \mathrm{SD}$ (Range).

The patients were also asked to respond to a simple questionnaire to determine the presence of psychogenic symptoms, sensitivity to noise, or vertigo symptoms during the scan.

The inclusion criteria were enrollment by the IRB with a head MRI scan and a completed questionnaire. The following exclusion criteria were used to classify the cases: fever, hypertension, cerebral perfusion, brain tumor at thalamus, claustrophobia, pregnancy, and anesthetized patients.

\subsection{Equipment}

All of the clinical MRI scans were acquired with a 1.5T system (Signa, General Electric Healthcare (GE); Milwaukee, WI, USA), a 3.0-T HDx system (GE), and a 3.0-T Philips scanner(Philips Achieva; Philips Medical Systems, Best, The Netherlands) using a standard clinical pulse sequence protocol. Table 1 shows the distribution of MRI types and subjects for head MRI scans. The RF transmission-receiving head coils were configured in an eight-channel regularphased array coil for the 3.0-T scans and as a one-channel 
coil for the 1.5-T scans. The RF power amplifiers (AMP) used for each scanner were as follows: GE 1.5 T: $16 \mathrm{~kW}$, GE $3.0 \mathrm{~T}$ : $35 \mathrm{~kW}$, and Philips Achieva 3.0 T: $25 \mathrm{~kW}$. During the scans, the RF power stayed below the maximum SAR of 3 watts per kilogram of head weight $\left(\mathrm{W} \mathrm{kg}^{-1}\right.$ ) (to the head), which is based on the FDA-proposed MRI safety guidelines. The SAR values depend on the strength of the static magnet field, RF AMP, repetition time (TR), echo train length (ETL), and number of excitations (NEX). To diminish the effect of $1.5 \mathrm{~T}$ and 3.0 T, the brain MRI protocol was properly adjusted (Table 2). The scan time (24 minutes) for the 3.0-T units (GE and Philips) were similar with a slightly lower scan time than that of the 1.5-T unit (28 minutes). Regarding age and HR, the subjects for the 1.5-T and 3.0-T units were analyzed separately.

We used a Veris MR-compatible vital signs monitoring system (MEDRAD, Indianola, PA, USA) to measure $\mathrm{O}_{2}$ saturation and HR during MRI scans of the brain. This system is designed for application in MRI environments up to 3.0 $\mathrm{T}$, and monitoring is designed for attachment to the index finger. Vital signs were checked at four stages at 10minute intervals during the scan: before and at the beginning, middle, and end of the MRI scan. The HR of each subject was analyzed over the course of the four-stage MRI process.

Body temperature was measured using an infrared tympanic thermometer (Thermoscan IRT 4520; accuracy range \pm 0.2 ; Braun, Kronberg, Germany). We checked both tympanic membrane temperatures of the subjects before the MRI scan and immediately after the scan (within $1 \mathrm{~min}$ ). If a difference between the two temperatures was evident, then the average temperature was recorded. The patient's vital signs were monitored continuously.

\subsection{MRI Scanning}

Subjects were in the supine position on an MRI table and were instructed to remain still during the imaging study. Subjects wore headphones to communicate with the researcher and attenuate the noise of the scanner; they were also provided with a hospital gown and were covered with a light blanket to keep warm. The MRI protocol sequence was as follows (Table 2): 1) axial T2 fast spin echo (FSE), 2) axial T1 FSE, 3) axial T2 fluid-attenuated inversion recovery (FLAIR), 4) axial diffusion, 5) sagittal, 6) coronal T2 FSE, 7) axial T1 FLAIR, 8) sagittal T1 FLAIR, and 9) coronal T1 FLAIR. This protocol was applied to all subjects who underwent brain MRI. A routine clinical brain MR protocol was performed, with a scan time of $\sim 24$ minutes for the 3.0T units (HDx, GE, and Philips Achieva). The scan time was slightly longer for the 1.5-T unit (Signa, GE) using a similar protocol. The temperature and humidity of the MRI rooms were maintained at $20 \pm 1^{\circ} \mathrm{C}$ and $40 \pm 2 \%$, respectively.
The main factor affecting body temperature rise is the SAR. Unfortunately, we do not have a device to measure the SAR value directly. Instead, SAR values were estimated automatically by the scanner-displayed SAR sequence, based on the input volume (70 kg in weight for all patients). It is not easy to compare the SAR values of the three types of MRI systems used in this study due to the different operation systems and characteristics (e.g., strength of the static magnetic field, RF AMP, repetition time, ETL, and NEX). SAR is a complex function of numerous variables that include the frequency, repetition time, type of RF coil used, configuration of the anatomic region exposed, and orientation of the body to the field vectors, as well as other factors (6, $12,14,16,25)$.

\subsection{Statistical Analysis}

The differences in the pre-scan and post-scan temperature dependence on magnetic field strength and resulting vital sign response (based on the subject's age, sex, BMI, oxygenation saturation, and HR), were determined from linear and multiple regressions using PASW ver. 18 (SPSS, IBM, NY, USA). Pearson's correlation coefficient analysis was used to evaluate correlations between $\mathrm{HR}$, HR patterns, and body temperature increase. A t-test was used to compare the difference between HR pattern types (up and down patterns) and difference in the body temperature between 3.0-T and 1.5-T systems during the MRI procedures. For all statistics, 95\% confidence intervals (CIs) were calculated and $\mathrm{p}$ values were considered significant at the level of 0.05 .

\section{Results}

\subsection{Association Between Body Temperature and MRI System}

Figure 1 shows that the mean and standard deviation (SD) values of body temperature increase were dependent on the RF power deposited. The maximum temperature increase (its mean and SD) was $1.1(0.72 \pm 0.20)^{\circ} \mathrm{C}$ and 0.9 (0.6 $\pm 0.10){ }^{\circ} \mathrm{C}$ for the 3.0 -T ( $25 \mathrm{~kW}$ and $35 \mathrm{~kW}$ ) and 1.5 -T systems $(16 \mathrm{~kW})$, respectively. Thus, there was a significant association between body temperature increase and the RF power deposited during an MRI scan $(\mathrm{P}=0.049)$; higher magnetic field strengths increased SAR values possibly contributing to higher body temperatures.

\subsection{Association Between Age and Body Temperature Increase During MRI}

It was reported that the body temperature increase during an MRI scan was correlated with the age of the patient $(12,36)$. In this study, we evaluated the relationship between age and body temperature rise as a function of 
Table 2. Characteristics of the Sequence Parameters During Brain MRI

\begin{tabular}{|c|c|c|c|c|c|c|}
\hline Field Strength & Pulse Sequence & TR (ms) & TE (ms) & ETL & Duration (min) & Est. SAR (W/kg) \\
\hline 1.5T/3.0T & AX T2 FSE & $4000 / 3000$ & $102.8 / 80$ & $11 / 6$ & $2: 32 / 2: 21$ & $1.17 \mid 1.6$ \\
\hline 1.5T/3.0T & AX T1 FSE & $450 / 360$ & $7.4 / 10$ & $8 / 7$ & $2: 07 / 2: 38$ & $1.63 / 2.6$ \\
\hline 1.5T/3.0T & AX T2 FLAIR & $8800 / 150$ & $150 / 125$ & $6 / 17$ & $3: 31 / 2: 37$ & $0.67 / 1.03$ \\
\hline $1.5 \mathrm{~T} / 3.0 \mathrm{~T}$ & AX Diffusion & $14000 / 9000$ & $112 / 60$ & & $2: 03 / 1: 21$ & $0.04 / 0.32$ \\
\hline 1.5T/3.0T & SAG T1 FSE & $600 / 450$ & $8 / 10$ & $9 / 6$ & $2: 20 / 2: 48$ & $1.21 / 2.2$ \\
\hline $1.5 \mathrm{~T} / 3.0 \mathrm{~T}$ & COR T2 FSE & $4000 / 3000$ & $105 / 80$ & $12 / 6$ & $2: 57 / 2: 32$ & $1.09 / 1.7$ \\
\hline $1.5 \mathrm{~T} / 3.0 \mathrm{~T}$ & AX T1 FLAIR & $2800 / 2500$ & $25 / 9.8$ & $10 / 5$ & $2: 59 / 3: 12$ & $1.45 / 2.5$ \\
\hline $1.5 \mathrm{~T} / 3.0 \mathrm{~T}$ & SAG T1 FLAIR & $2800 / 2500$ & $25 / 9.8$ & $10 / 6$ & $2: 38 / 2: 43$ & $1.35 / 2.4$ \\
\hline $1.5 \mathrm{~T} / 3.0 \mathrm{~T}$ & COR T1 FLAIR & $2800 / 2500$ & $25 / 9.8$ & $10 / 5$ & $2: 36 / 2: 40$ & $1.45 / 2.5$ \\
\hline
\end{tabular}

Abbreviations: AX, axial;SAG, sagittal; COR, coronal; TR, repetition time; TE, echo time; ETL, echo train length; FOV, field of view; FSE, fast spin echo; FLAIR, fluid-attenuated inversion recovery; Est. SAR, estimated SAR; based on the entered weight (70 kg), was obtained with consent from a healthy male adult donor; each pulse sequence parameter was compared between the GE systems (1.5 T Signa and 3.0 T HDx), due to the same MR system's inner workings; 3.0 T Achieva systems could not be identified with Est. SAR

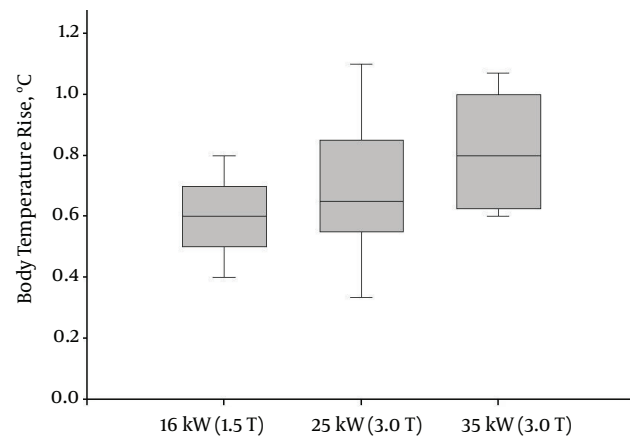

Figure 1. Mean body temperature increase as a function of magnetic resonance imaging (MRI) RF power deposited after the brain MRI protocol sequence. The mean values are indicated as the inner line of the boxes with standard error bars. Mean body temperature increase categorized as MRI magnetic resonance field strength indicates statistically significant differences $(\mathrm{P}<0.05)$.

the RF power deposited. Our results indicated a slight difference between 1.5- and 3.0-T fields. Regarding 1.5 T, there was no significant correlation between age and body temperature rise $(r=0.07, \mathrm{P}=0.29)$. However, the body temperature increase observed with 3.0-T MRIs was clearly correlated with age, with a higher increase in older subjects ( $\mathrm{r}$ $=0.45, \mathrm{P}=0.002)$. Figure 2 shows the correlation between body temperature rise and age.

\subsection{Association Between HR and Body Temperature Increase During MRI Scan}

Generally, HR can display an irregular pattern. Moreover, HR can demonstrate a more irregular pattern if the patients are placed in a noisy, confined space with a limited field of vision during the MRI. Therefore, according to a difference in the change in HR during the MRI procedure, the relationship with body temperature is determined.

The HR was checked at the beginning, middle, and end of the MRI scan. Three HR values were recorded at each checkpoint. These three values were averaged to calculate the mean HR value. Table 3 shows the results of the HR and temperature change during the MRI. The regression between the average HR value for each patient and temperature rise indicated no significant correlation at $1.5 \mathrm{~T}(\mathrm{P}=$ $0.98 ; \mathrm{r}=0.09)$ or $3.0 \mathrm{~T}(\mathrm{P}=0.98 ; \mathrm{r}=0.08)$.

We then classified each HR pattern as "up" or "down" according to the change in HR during the MRI procedure. That is, if initially the HR was faster than the HR obtained at the end of the MRI procedure (the HR had slowed), then the HR pattern exhibited a downward tendency or "down" pattern. Conversely, if the initial HR was slower than the HR obtained at the end of the MRI, then the HR pattern exhibited an upward tendency or "up" pattern. Figure 3 shows a comparison of the up and down patterns of the HR and the corresponding body temperature response. Unlike the direct comparison between HR and body temperature increase, in this case, the body temperature increase depended on the type of HR pattern (up or down) during the MRI study ( $\mathrm{p}=0.04$ at $1.5 \mathrm{~T}$, and $\mathrm{P}=0.03$ at $3.0 \mathrm{~T}$; $\mathrm{t}$ test). That is, when the HR pattern during MRI scan was up (HR was fast), a smaller increase in body temperature was observed. Conversely, when the HR pattern was down (HR was slow), a greater increase in body temperature was evident. Additionally, the HR pattern may be classified as downward or upward during the MRI procedure. The difference in HR over the course of the scan was within \pm 5 in all subjects. Figure 4 shows the relationship between the increase in body temperature and the difference in HR dur- 
A

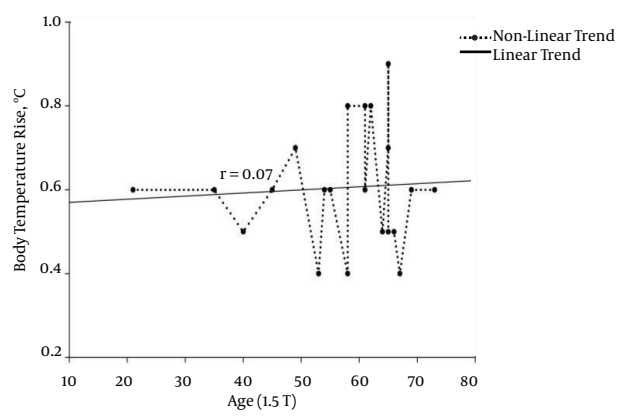

B

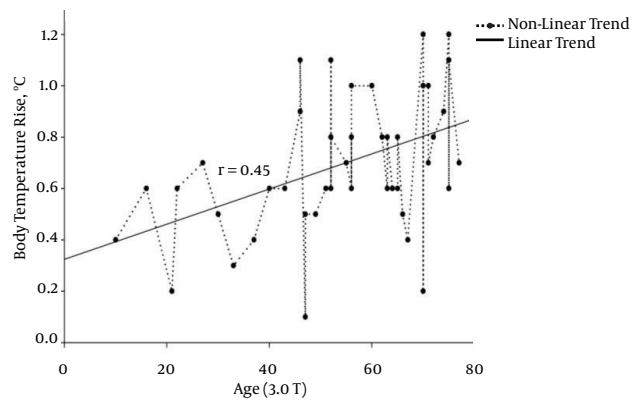

Figure 2. Distribution chart and linear regression graph of the age-dependent increase in body temperature during 1.5-T $(P=0.29)(A)$ And 3.0-T $(P=0.002)(B)$ MRI scans. The plot shows body temperature increase during the MRI process. Body temperature increase during 3.0-T MRI procedures was correlated with subject age as shown in (B).

Table 3. Multivariate Regression Model for Estimating Body Temperature Rise During Brain MRI Based on Patient Characteristics and MRI Machine ${ }^{\mathrm{a}}$

\begin{tabular}{|c|c|c|c|c|}
\hline \multirow[t]{2}{*}{ Variables } & \multirow[t]{2}{*}{$\beta$ (Standardized Coefficient) } & \multicolumn{2}{|c|}{ 95\% confidence interval $(\mathrm{CI})$} & \multirow[t]{2}{*}{ PValue } \\
\hline & & Lower & Upper & \\
\hline Sex & -0.07 & -0.13 & 0.07 & 0.55 \\
\hline Age, $y$ & 0.28 & 0.00 & 0.00 & 0.01 \\
\hline BMI & -0.11 & -0.02 & 0.01 & 0.29 \\
\hline $\begin{array}{l}\text { HR Difference } \\
\text { During MRI }^{\mathbf{c}}\end{array}$ & -0.55 & -0.04 & -0.01 & 0.01 \\
\hline MRI Scanner Type $^{d}$ & 0.21 & -0.02 & 0.01 & 0.19 \\
\hline
\end{tabular}

Abbreviations: BMI, body mass index; HR, heart rate

${ }^{a}$ Results were statistically analyzed using multiple regression.

${ }^{\mathrm{b}}$ HR patterns are up pattern and down pattern.

${ }^{\mathrm{c}} \mathrm{HR}$ difference: linear value

${ }^{\mathrm{d}}$ MRI scanners were 1.5-T MRI and 3.0-T MRI.

ing the MRI procedure $(\mathrm{r}=-0.42, \mathrm{P}=0.05$ at $1.5 \mathrm{~T}$, and $\mathrm{r}=$ $-0.44, \mathrm{P}=0.002$ at $3.0 \mathrm{~T})$. That is, generally, the body temperature rise increased sharply when the HR tendency was downward instead of upward during the MRI procedure.

\subsection{Association Between Temperature Increase and Age, and $H R$}

The relationship between age and body temperature was related to HR and changes in HR pattern during the MRI study. To determine the effects on body temperature as a function of age, the subjects were divided into three categories: 10 to 39 years, 40 to 59 years, and older than 59 years. Figure 5 shows the relationship between the mean body temperature increase and HR pattern during the MRI procedure, according to age. An increase in the mean body temperature was observed in older subjects (the mean body temperature rises were $0.6,0.69$, and $0.75^{\circ} \mathrm{C}$ for subjects aged younger than 39 years, 40 to 59

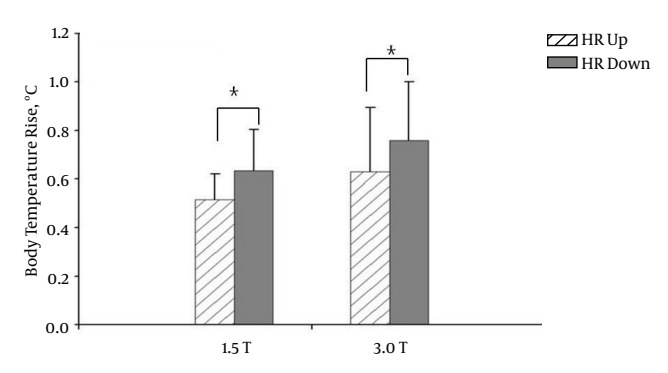

Figure 3. Bar graphs of mean body temperature increase $\left({ }^{\circ} \mathrm{C}\right)$ and heart rate (HR) as a function of MRI magnetic field strength. Error bars indicate standard deviations. A relationship between body temperature increase and change in HR over the course of MRI scan is apparent. For example, body temperature increase was higher if the initial HR (before MRI scan) was faster than that at the end of the MRI procedure. Vertical lines represent standard deviation. Star symbols *, indicate statistically significant differences. *; $\mathrm{P}<0.05$.

years, and older than 59 years, respectively, when the HR 
A

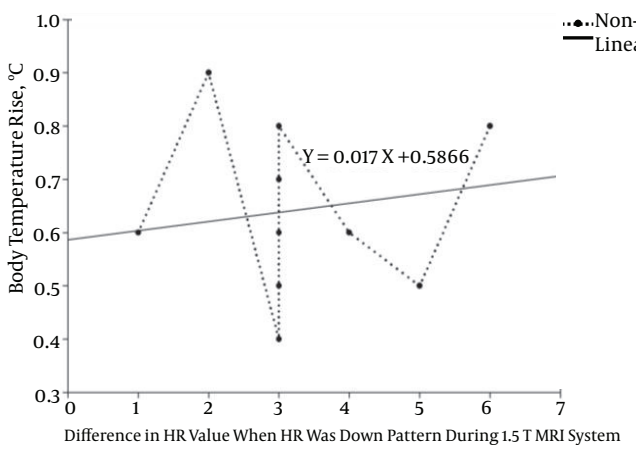

C

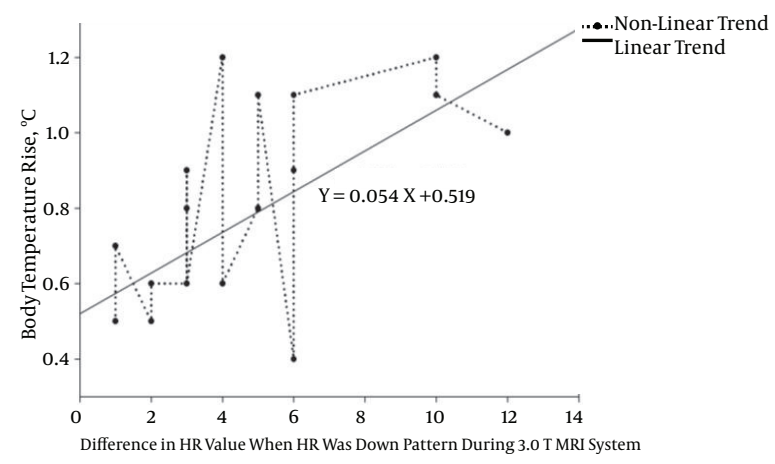

B

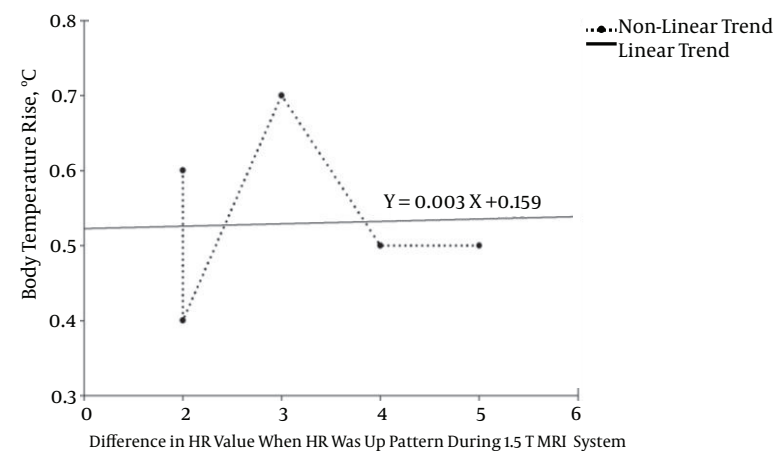

D

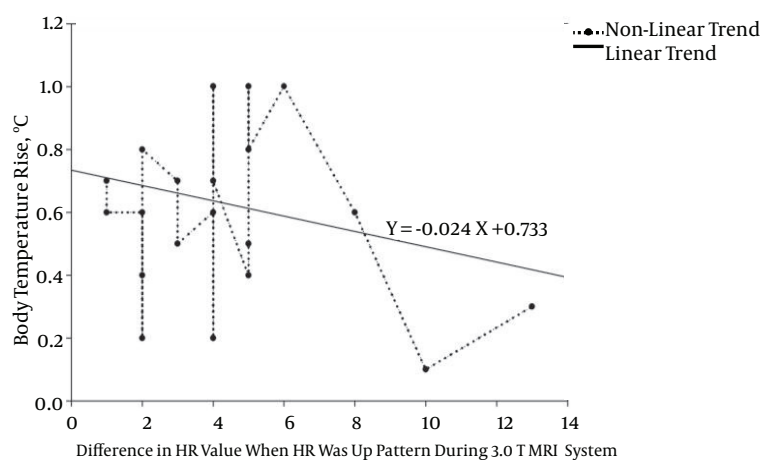

Figure 4. Distribution chart and linear regression graph of the relationship between the increase in body temperature and difference in HR during the MRI procedure (represented as a "down" or "up" pattern). A, a down pattern and B, an up pattern for $1.5 \mathrm{~T}$. C, a down pattern and D, an up pattern for $3.0 \mathrm{~T}$. Here, an up (down) pattern corresponds to a fast (a slow) in the HR over the course of the MRI scan. Each plot represents the body temperature increase of a single subject. The $\mathrm{x}$-axis values indicate the mean change in HR during the MRI scan. If the HR tendency is downward (indicating a slowing HR), then a greater increase in body temperature was observed ((A) and (C)). For an upward HR pattern (indicating a fasting HR), the increase in temperature was markedly less, as shown in (B) and (D).

Table 4. Distribution of Physiological Responses for Brain MRI ${ }^{\mathrm{a}}$

\begin{tabular}{|cc}
\hline Physiological Responses & Results \\
\hline Average HR, Mean \pm SD $(\mathbf{B P M})$ & \\
\hline Initial $(1.5 \mathrm{~T} / 3.0 \mathrm{~T})$ & $79.05 \pm 11.53 / 77.97 \pm 10.47$ \\
\hline Final $(1.5 \mathrm{~T} / 3.0 \mathrm{~T})$ & $77.85 \pm 10.97 / 78.10 \pm 11.05$ \\
\hline Average Temperature, $\mathbf{M e a n} \pm \mathbf{S D}\left({ }^{\circ} \mathbf{C}\right)$ & \\
\hline Initial $(1.5 \mathrm{~T} / 3.0 \mathrm{~T})$ & $36.67 \pm 0.21 / 36.61 \pm 0.27$ \\
\hline Final $(1.5 \mathrm{~T} / 3.0 \mathrm{~T})$ & $37.27 \pm 0.17 / 37.33 \pm 0.22$ \\
\hline Temperature Change $\left({ }^{\circ} \mathbf{C}\right)$ & $0.1-1.1$ \\
\hline
\end{tabular}

Abbreviations: HR, heart rate; SD, standard deviation; BPM, beat per minute ${ }^{\mathrm{a}}$ Initial and final indicates before and after brain MRI.

displayed a downward pattern during the MRI procedure). Furthermore, the relationship between body temperature rise and age showed a greater effect on 3.0 T (the mean body temperature rises were $0.6,0.73$, and $0.80^{\circ} \mathrm{C}$ for subjects aged younger than 39 years, 40 to 59 years, and older than 59 years, respectively, when the HR showed a downward pattern during the MRI procedure). Thus, the HR data indicated that older patients have a decreased HR compared with younger patients.

\subsection{Association Between Temperature Rise and Physiological} Parameters (Weight or BMI, Gender, and O2 Saturation)

We assumed that the thermoregulatory responses would depend on the specific heat capacity and body surface area. However, the results indicated that body weight did not affect body temperature increase during an MRI $\operatorname{scan}(\mathrm{r}=0.13, \mathrm{P}=0.43$ at $1.5 \mathrm{~T} ; \mathrm{r}=-0.1, \mathrm{P}=0.46$ at $3.0 \mathrm{~T})$. There was no correlation observed between the BMI and body temperature increase in the 1.5- $\mathrm{T}$ unit $(\mathrm{r}=0.012, \mathrm{P}=0.60)$, but a weak correlation was observed in the 3.0-T units ( $\mathrm{r}=$ $0.05, \mathrm{P}=0.12$ at $3.0 \mathrm{~T}$ ).

We also assumed that the thermoregulatory responses would depend on gender/sex. Generally, male subjects 

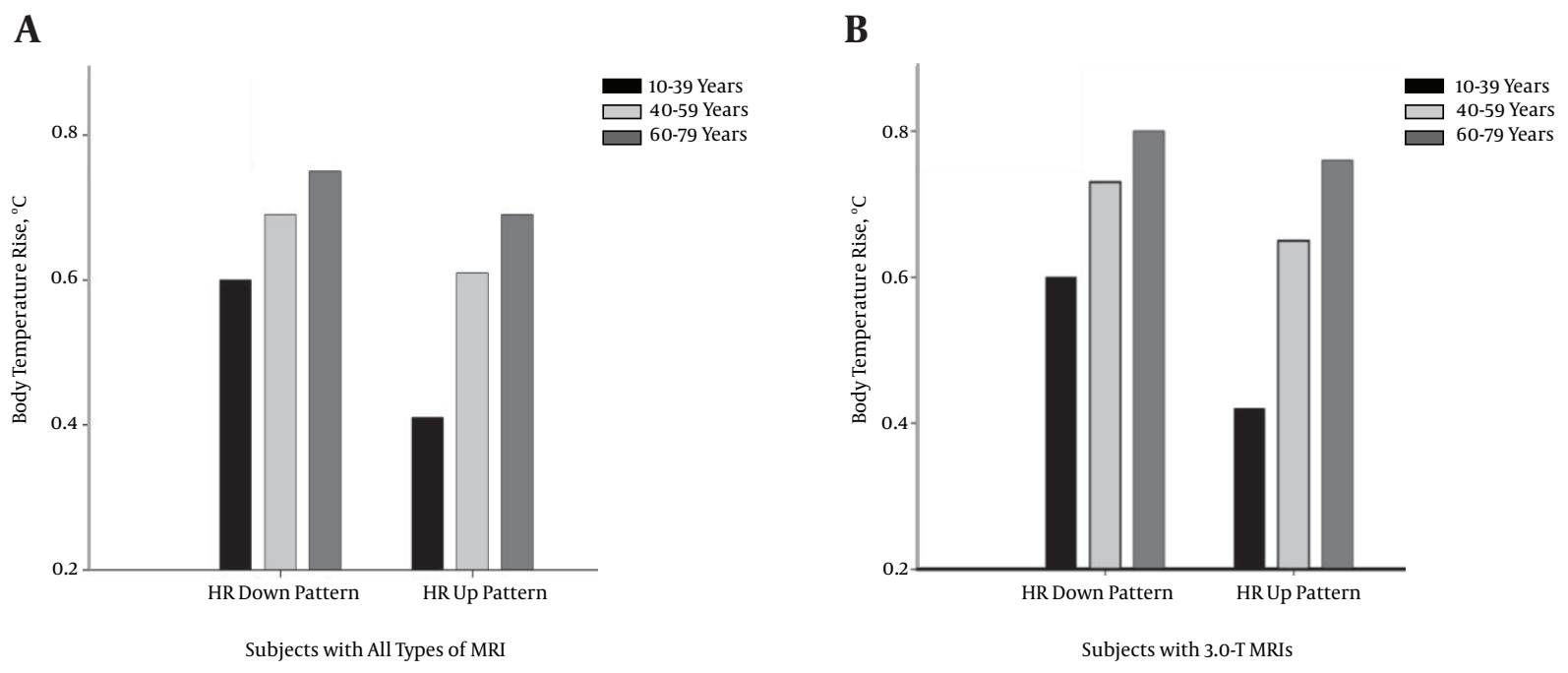

Figure 5. Dependence of the increase in mean body temperature on the change in HR, according to the following age categories: 10 to 39,40 to 59 , and 60 to 79 years. A, Body temperature increase of all subjects treated with various types of MRIs. B, Body temperature increase of subjects treated with a 3.0-T MRI.

showed a greater mean body temperature rise ( 0.66 and $0.70^{\circ} \mathrm{C}$ for $1.5 \mathrm{~T}$ and $3.0 \mathrm{~T}$, respectively) than female subjects ( 0.59 and $0.66^{\circ} \mathrm{C}$ for $1.5 \mathrm{~T}$ and $3.0 \mathrm{~T}$, respectively). However, the male subjects were older than the female subjects (mean female age: 54.6 and 51.4 years for $1.5 \mathrm{~T}$ and $3.0 \mathrm{~T}$, respectively; mean male age: 60.8 and 55.9 years for $1.5 \mathrm{~T}$ and 3.0 T, respectively). However, a weak correlation ( $\mathrm{r}=-0.22$, $\mathrm{P}=0.11$ at $1.5 \mathrm{~T} ; \mathrm{r}=-0.13, \mathrm{P}=0.33$ at $3.0 \mathrm{~T}$ when coded as male (code1) and female (code 2)) was observed between sex and body temperature increase during MRI, but without statistically significant results.

No significant correlations were found between $\mathrm{O} 2$ saturation and body temperature rise during MRI $(\mathrm{r}=-0.02, \mathrm{P}$ $=0.93$ at $1.5 \mathrm{~T} ; \mathrm{r}=-0.03, \mathrm{P}=0.83$ at $3.0 \mathrm{~T}$ ).

\subsection{Body Temperature Increase and Cognitive Function}

We assumed that the thermoregulatory system was dependent on the psychological condition of the subjects, as well as the physiological response of the body to RF heat stress. Because the patients were placed in a noisy, long, confined space, with a limited field of vision during the MRI procedure, it was expected that they felt uncomfortable. All of the subjects were asked about how they felt during the MRI procedure. Their comments were classified based on the degree of the response, which varied from calm/sleepiness to anxiousness/claustrophobia. However, no relationship was identified between psychological condition and body temperature rise. Fourteen (67\%) patients were placid; six (28\%) felt anxious; and one (5\%) experienced claustrophobia during the 1.5-T MRI scan. During the
3.0-T MRI scan, 1 (2\%) fell asleep; 1 (2\%) had relief; 25 (52\%) were placid; and 21 (44\%) felt anxious. The correlation between mental state and body temperature rise was $r=0.33$ for $1.5 \mathrm{~T}$, and $\mathrm{r}=0.29$ for $3.0 \mathrm{~T}$.

\section{Discussion}

The purpose of this study was to investigate the relationship between body temperature rise and the RF power deposited during a routine clinical head MRI examination, and to determine the correlation between this effect and the physiological response of the body. Our results showed that measurable heating of the body occurred during routine head MRI scanning despite maintaining a magnet temperature of $20 \pm 1^{\circ} \mathrm{C}$ and holding the relative humidity at $<40 \%$. Note that only a single cotton blanket was placed over the patient to reduce passive heat loss.

The increase in body temperature caused by absorbed RF energy during MR procedures depends on various physiological, physical (MR system), and environmental factors $(12,37)$. The amount of heat generated depends on the amount of RF energy absorbed during MR acquisition, because most RF energy is converted into heat $(13,15$, 34). As SAR values increased with the main magnetic field strength, the body temperature rise was much higher for the Tesla scans due to the amount of RF energy absorbed $(12,16,37)$. In our study, the maximum increase in body temperature exceeded $1.1^{\circ} \mathrm{C}$ after 30 minutes of scanning at 3.0 $\mathrm{T}$ (1 patient). In contrast, only 1 of 21 patients (4.7\%) who underwent 1.5-T scans exhibited a body temperature increase of up to $0.9^{\circ} \mathrm{C}(25 \%$ of those undergoing 3.0-T scans 
[12 of 49 patients] demonstrated such a temperature increase). As SAR values increased with the main magnetic field strength, body temperature rise was much higher for the 3.0-T scans due to the amount of RF energy absorbed compared with the low-field strength scans at $1.5 \mathrm{~T}(15,38)$.

Our investigation of the physiological response to RF heating indicated a correlation between increasing age and body temperature. The maximal $\mathrm{HR}\left(\mathrm{HR}^{\mathrm{max}}\right)$ dependence on age $(29,39)$ and the stroke volumes are given by the following Equation 1 :

$H R_{\max }=220-$ age

Stroke Volume $=\frac{\text { Cardiac Output }}{H R}$

According to the relationship between age and HR, maximum HR value and cardiac output volume decrease with age. Several studies have reported variation in the blood circulation time as a result of complex factors. The function of the left ventricular ejection time (LVET) and HR is expected to degenerate with age $(29,40,41)$. Therefore, circulation time increases with age $(29,39,41,42)$.

Body heat resulting from RF radiation exposure is dissipated by means of convection, conduction, radiation, and evaporation $(13,37)$. A physiological response is associated with age; a decrease in aerobic power, evaporative sweat rate, cardiovascular fitness of the maximal HR, blood flow, and tolerance to heat stress was observed in older individuals $(12,37,42,43)$. Because increase of age was associated with a decrease in the body's thermoregulatory response, heating caused by exposure to RF radiation was higher for older patients. The results of the relationship between age and body temperature rise were $r=0.07$ and $\mathrm{P}=0.29$, indicating no correlation for 1.5-T scans. However, in patients who underwent 3.0-T MRI scans, there was a clear relationship between age and body temperature rise $(r=0.45$ and $\mathrm{P}=0.002$ ). Older subjects experienced a higher temperature increase. Therefore, age may be related to the HR value $(24,32,44)$. The 3.0-T scanners had twice the magnetic field strength and a much higher SAR value than the 1.5-T systems. Additionally, the distribution of subject age in this study was larger for the 3.0-T group $(53.95 \pm 12.96)$ than the1.5-T group (56.47 \pm 9.56 ), which allowed observation of this relationship (a greater increase in body temperature for the 3.0-T scans).

Figure 5 shows the association between body temperature increase and HR pattern according to age category. The relationship between temperature and HR pattern according to the age of the patients undergoing the 3.0$\mathrm{T}$ procedure (Figure $5 \mathrm{~B}$ ) was clearer than that for subjects assessed with other types of MRIs (Figure 5A) due to the marked temperature change in response to RF exposure. The results also showed that the dependence on the change in $\mathrm{HR}(\mathrm{r}=-0.42, \mathrm{P}=0.05$ at $1.5 \mathrm{~T}$, and $\mathrm{r}=-0.44, \mathrm{P}=$ 0.002 at $3.0 \mathrm{~T})$ was greater than that on the age $(\mathrm{r}=0.06, \mathrm{P}$ $=0.77$ at $1.5 \mathrm{~T}$, and $\mathrm{r}=0.41, \mathrm{P}=0.02$ at $3.0 \mathrm{~T}$ ) of the subject. The HR pattern during the MRI study was the most sensitive physiological response evaluated in this study.

Human blood circulation is transferred to peripheral tissue with energy supplied by cardiomotility, which may also serve as a thermoregulatory system for the body. Blood circulation may lower localized temperature increase in the body by eliminating water transfer from the blood to the outside (18).

A previous study of the influence of increased temperature on the blood perfusion rate indicated that to remove the excess heat produced when the temperature exceeds a certain level, as detected at the hypothalamus $(33,36)$, blood perfusion to the skin is increased via vasodilation. However, thermoregulation via sweating was slight in the previous study (36).

Our findings indicated that age and HR pattern were the physiological responses related to heating; however, patient sex, weight, $\mathrm{BMI}, \mathrm{O}_{2}$ saturation, and psychological response did not correlate with the rise in body temperature. Therefore, cardiac output or blood circulation (blood flow rate and blood volume) is a major factor that controls body temperature. We hypothesized that body temperature rise is affected by body type or $\operatorname{BMI}(26,27,32)$.

Based on FDA(2004) findings, we present the following Equation 2 for SAR:

$S A R=\sigma|E| \frac{2}{2 P_{t}}$

Where $\sigma$ is the electrical conductivity, $\mathrm{E}$ is the amplitude of the electric field strength, and $\rho_{\mathrm{t}}$ is the tissue density in $\mathrm{kg} \mathrm{m}^{-3}$ (28). We speculated that the higher the density of the subject, the greater the increase in body temperature. Therefore, an increase in the BMI should be accompanied by an increase in the ability of the body to absorb as well as dissipate heat. However, our data showed no significant correlation with weight or BMI.

Over the course of a 30-minute MRI scan, the body gradually loses its ability to maintain its regulatory system (homeostasis) $(11,37)$. Especially in patients with impaired cerebral perfusion or those who are pregnant, continuous elevations in RF exposure may not be appropriate due to an improper thermoregulatory response (12). In our study, two patients were anesthetized, and two were pregnant while undergoing MRI. Anesthetized patients are thermodynamically fragile, so their body temperature increased more during the MRI study. In addition, the pregnant patients complained of a stifled feeling with the rise in body temperature, which eventually led to abandonment of MRI. We attributed this response to the confined 
nature of the MRI system.

Although the results of the anesthetized and pregnant patients are not reflected in the outcome, they provide a good example of thermodynamically fragile subjects. Poor heat dissipation in body parts with a higher water density, such as the testicles and eyes, will result in a relatively rapid temperature increase $(7,32,37)$.

Another vital response was related to the body temperature rise during an MRI procedure in patients with impaired cerebral perfusion due to stroke, tumors, or arteriovenous malformation $(12,26)$. Although some of the brain-MRI scanned patients (56.5\%) had a tumor, according to the radiologist's interpretation of the images, no significant change in temperature was evident (linear regression, $\mathrm{P}=0.1$ ).

The response of the body to RF-related heating depends on multiple physiological (e.g., thermoregulatory system, hypertension, diabetes, etc.) responses $(33,37)$ and MR factors (e.g., duration of exposure, RF power AMP, and sequence type)(22). Our results showed a maximum temperature rise of $1.1^{\circ} \mathrm{C}$ for a 3.0-T MRI scan; thus, the higher the RF power deposited, the greater the increase in body temperature. As the magnetic field strength is increased to provide higher contrast in MRI, MRI examiners are encouraged to consider the risks associated with sudden increases in temperature, which could jeopardize the health of the patient, particularly those with an impaired thermoregulatory system, including the elderly. Although the adverse health effects secondary to RF exposure remain unknown, they may not be so minor as to be ignored (21).

We know that the effects of environmental changes on body heat response are decided by several physiological responses. Nevertheless, this study focused on the elevation of temperature via limited variables (RF Amp, HR, age, and BMI) during MRI performance.

In conclusion, the higher the RF power deposited and the greater the decrease in HR, the greater the increase in body temperature resulting from MRI procedure. Our findings indicated that the change in HR was a major factor in the degree of thermoregulatory response to RF heating.

In normal individuals, relatively high magnetic field exposure may not be an issue due to functional thermoregulatory responses $(10,12,14,37)$. However, many patients who undergo MRI procedures are impaired (fever, hypertension, impaired cerebral perfusion, brain tumor, and decreased HR). Additionally, particular medications (muscle relaxers, sedatives, beta-blockers, and diuretics) have a synergistic effect with heating caused by RF radiation exposure (37). Thus, MRI examiners must strictly follow FDA and international commission on non-ionizing radiation protection (ICNIRP) guidelines $(9,45,46)$.

This study had several limitations. First, body tempera- ture was measured not during the brain MRI scan but after the scan. An MRI-compatible thermometer was not available to measure the body temperature during the MRI procedure; thus, the peak increase in body temperature could not be checked by RF pulse. Instead, an infrared tympanic thermometer was used to monitor changes in the body temperature immediately after brain MRI.

It is not easy to compare SAR values of the three types of MRI systems used in this study due to different inner workings (different operation systems) and characteristics (e.g., strength of the static magnet field, RF AMP, repetition time, ETL, and NEX). The main factor affecting body temperature rise is SAR. Unfortunately, we do not have a device to measure SAR value directly. However, SAR values were estimated automatically by scanner-displayed SAR sequence based on the input volume (70-kg weight for all patients).

Our survey data showed a weak correlation between mental state and temperature rise. Mental state is a subjective response from the questionnaire. So, further studies with more subjects is needed to confirm the association.

\section{Footnotes}

Authors' Contributions: Myeong Seong Kim was responsible for all parts of the study and writing of the manuscript.

Financial Disclosure: None declared. Funding/Support: None declared.

\section{References}

1. Institute of Physics Report. MRI and the Physical Agents (EMF) Directive London (UK): NHS Foundation Trust and King's College London; 2008. Available from: http://www.iop.org/publications/iop/ 2008/file_38215.pdf.

2. Jung W, Zvereva V, Hajredini B, Jackle S. Initial experience with magnetic resonance imaging-safe pacemakers : a review. J Interv Card Electrophysiol. 2011;32(3):213-9. doi: 10.1007/s10840-011-9610-0. [PubMed: 21993594].

3. Hinton DP, Wald LL, Pitts J, Schmitt F. Comparison of cardiac MRI on 1.5 and 3.0 Tesla clinical whole body systems. Invest Radiol. 2003;38(7):436-42. doi: 10.1097/01.RLI.0000067489.31556.70. [PubMed: 12821858].

4. Tanenbaum LN. Clinical $3 T$ MR imaging: mastering the challenges. Magn Reson Imaging Clin N Am. 2006;14(1):1-15. doi: 10.1016/j.mric.2005.12.004. [PubMed: 16530631].

5. Kuhl CK, Traber F, Schild HH. Whole-body high-field-strength (3.0-T) MR Imaging in Clinical Practice. Part I. Technical considerations and clinical applications. Radiology. 2008;246(3):675-96. doi: 10.1148/radiol.2463060881. [PubMed: 18309012].

6. Collins CM, Smith MB. Calculations of B(1) distribution, SNR, and SAR for a surface coil adjacent to an anatomically-accurate human body model. Magn Reson Med. 2001;45(4):692-9. [PubMed: 11283998].

7. Formica D, Silvestri S. Biological effects of exposure to magnetic resonance imaging: an overview. Biomed Eng Online. 2004;3:11. doi: 10.1186/1475-925X-3-11. [PubMed: 15104797]. 
8. Simmons A, Hakansson K. Magnetic resonance safety. Methods Mol Biol. 2011;711:17-28. doi: 10.1007/978-1-61737-992-5_2. [PubMed: 21279596].

9. Food and Drug Administration . Criteria for significant risk investigations of magnetic resonance diagnostic devices. Washington DC: Food and Drug Administration; 2003.

10. Chakeres DW, Kangarlu A, Boudoulas H, Young DC. Effect of static magnetic field exposure of up to 8 Tesla on sequential human vital sign measurements. J Magn Reson Imaging. 2003;18(3):346-52. doi: 10.1002/jmri.10367. [PubMed: 12938131].

11. Weintraub MI, Khoury A, Cole SP. Biologic effects of 3 Tesla (T) MR imaging comparing traditional $1.5 \mathrm{~T}$ and $0.6 \mathrm{~T}$ in 1023 consecutive outpatients. J Neuroimaging. 2007;17(3):241-5. doi: 10.1111/j.15526569.2007.00118.x. [PubMed: 17608910].

12. Shellock FG, Crues JV. MR procedures: biologic effects, safety, and patient care. Radiology. 2004;232(3):635-52. doi: 10.1148/radiol.2323030830. [PubMed: 15284433].

13. Yang M, Christoforidis G, Abduljali A, Beversdorf D. Vital signs investigation in subjects undergoing MR imaging at 8T. AJNR Am J Neuroradiol. 2006;27(4):922-8. [PubMed: 16611792].

14. Dempsey MF, Condon B. Thermal injuries associated with MRI. Clin Radiol. 2001;56(6):457-65. doi: 10.1053/crad.2000.0688. [PubMed: 11428795].

15. Park SM, Nyenhuis JA, Smith CD, Lim EJ, Foster KS, Baker KB. Gelled versus nongelled phantom material for measurement of MRI-induced temperature increases with bioimplants. Magnetics, IEEE Transactions. 2003;39(5):3367-71.

16. Baker KB, Tkach JA, Nyenhuis JA, Phillips M, Shellock FG, GonzalezMartinez J, et al. Evaluation of specific absorption rate as a dosimeter of MRI-related implant heating. J Magn Reson Imaging. 2004;20(2):315-20. doi: 10.1002/jmri.20103. [PubMed: 15269959].

17. Martin AJ, Baek B, Acevedo-Bolton G, Higashida RT, Comstock J, Saloner DA. MR imaging during endovascular procedures: an evaluation of the potential for catheter heating. Magn Reson Med. 2009;61(1):45-53. doi: 10.1002/mrm.21817. [PubMed: 19097197].

18. Gonzalez-Alonso J, Teller C, Andersen SL, Jensen FB, Hyldig T, Nielsen B. Influence of body temperature on the development of fatigue during prolonged exercise in the heat. J Appl Physiol (1985). 1999;86(3):1032-9. [PubMed: 10066720].

19. Hirata A, Asano T, Fujiwara O. FDTD analysis of human body-core temperature elevation due to RF far-field energy prescribed in the ICNIRP guidelines. Phys Med Biol. 2007;52(16):5013-23. doi:10.1088/00319155/52/16/020. [PubMed: 17671350].

20. Lee JW, Kim MS, Kim YJ, Choi YJ, Lee Y, Chung HW. Genotoxic effects of $3 \mathrm{~T}$ magnetic resonance imaging in cultured human lymphocytes. Bioelectromagnetics. 2011;32(7):535-42. doi: 10.1002/bem.20664. [PubMed: 21412810].

21. Lu H, Nagae-Poetscher LM, Golay X, Lin D, Pomper M, van Zijl PC. Routine clinical brain MRI sequences for use at 3.0 Tesla. J Magn Reson Imaging. 2005;22(1):13-22. doi: 10.1002/jmri.20356. [PubMed: 15971174].

22. Boss A, Graf H, Berger A, Lauer UA, Wojtczyk H, Claussen CD, et al. Tissue warming and regulatory responses induced by radio frequency energy deposition on a whole-body 3-Tesla magnetic resonance imager.J Magn Reson Imaging. 2007;26(5):1334-9. doi:10.1002/jmri.21156. [PubMed: 17969173].

23. Finelli DA, Rezai AR, Ruggieri PM, Tkach JA, Nyenhuis JA, Hrdlicka G, et al. MR imaging-related heating of deep brain stimulation electrodes: in vitro study. AJNR Am J Neuroradiol. 2002;23(10):1795-802. [PubMed: 12427641]

24. Wang C, Shen GX, Yuan J, Qu P, Wu B. Theoretical and experimental investigation of the relationship among SAR, tissues and radio frequencies in MRI. Phys Med. 2005;21(2):61-4. doi: 10.1016/S11201797(05)80020-1. [PubMed: 18348846].

25. Stralka JP, Bottomley PA. A prototype RF dosimeter for independent measurement of the average specific absorption rate (SAR) during MRI. J Magn Reson Imaging. 2007;26(5):1296-302. doi 10.1002/jmri.21141. [PubMed: 17969145].

26. Collins CM, Liu W, Wang J, Gruetter R, Vaughan JT, Ugurbil K, et al. Temperature and SAR calculations for a human head within volume and surface coils at 64 and $300 \mathrm{MHz}$. J Magn Reson Imaging. 2004;19(5):650-6. doi:10.1002/jmri.20041. [PubMed: 15112317].

27. Valentina H, Giovannetti G, Vanello N, Landini L, Santarelli MF. Numerical calculation of peak to average specific absorption rate on different human thorax models for magnetic resonance safety considerations. Appl Magn Reson. 2010;38(3):337-48.

28. Yeung CJ, Susil RC, Atalar E. RF safety of wires in interventional MRI: using a safety index. Magn Reson Med. 2002;47(1):187-93. [PubMed: 11754458].

29. Tanaka H, Monahan KD, Seals DR. Age-predicted maximal heart rate revisited. J Am Coll Cardiol. 2001;37(1):153-6. [PubMed: 11153730].

30. Watanabe J, Thamilarasan M, Blackstone EH, Thomas JD, Lauer MS. Heart rate recovery immediately after treadmill exercise and left ventricular systolic dysfunction as predictors of mortality: the case of stress echocardiography. Circulation. 2001;104(16):1911-6. [PubMed: 11602493].

31. Katzmarzyk PT, Janssen I, Ross R, Church TS, Blair SN. The importance of waist circumference in the definition of metabolic syndrome: prospective analyses of mortality in men. Diabetes Care 2006;29(2):404-9. [PubMed: 16443895].

32. Kenney WL. Physiological correlates of heat intolerance. Sports Med 1985;2(4):279-86. [PubMed: 2862686].

33. Hirata A, Sugiyama H, Fujiwara OE. Estimation of core temperature elevation in humans and animals for whole-body averaged SAR. Progress Electromagnetics Res. 2009;99:53-70.

34. Moshin SA, Sheikh NM, Saeed U. MRI induced heating of deep brain stimulation leads: effect of the air-tissue interface. Progress Electromagnetic Res. 2008;83:81-91.

35. Wang Z, Lin JC, Vaughan JT, Collins CM. Consideration of physiological response in numerical models of temperature during MRI of the human head. J Magn Reson Imaging. 2008;28(5):1303-8. doi: 10.1002/jmri.21556. [PubMed: 18972342].

36. Adair ER, Mylacraine KS, Allen SJ. Thermophysiological consequences of whole body resonant RF exposure $(100 \mathrm{MHz})$ in human volunteers. Bioelectromagnetics. 2003;24(7):489-501. doi: 10.1002/bem.10128. [PubMed: 12955754].

37. Shellock FG. Radiofrequency energy-induced heating during MR procedures: a review. J Magn Reson Imaging. 2000;12(1):30-6. [PubMed: 10931562].

38. van den Bergen B, van den Berg CA, Klomp DW, Lagendijk JJ. SAR and power implications of different RF shimming strategies in the pelvis for 7T MRI. J Magn Reson Imaging. 2009;30(1):194-202. doi: 10.1002/jmri.21806. [PubMed: 19557737].

39. Weissler AM, Harris WS, Schoenfeld CD. Bedside technics for the evaluation of ventricular function in man. Am JCardiol. 1969;23(4):577-83. [PubMed: 5781885].

40. Willems JL, Roelandt J, De Geest H, Kesteloot H, Joossens JV The left ventricular ejection time in elderly subjects. Circulation. 1970;42(1):37-42. [PubMed: 5425593].

41. Gellish RL, Goslin BR, Olson RE, McDonald A, Russi GD, Moudgil VK. Longitudinal modeling of the relationship between age and maximal heart rate. Med Sci Sports Exerc. 2007;39(5):822-9. doi: 10.1097/mss.ob013e31803349c6. [PubMed: 17468581].

42. Drinkwater BL, Horvath SM. Heat tolerance and aging. Med Sci Sports 1979;11(1):49-55. [PubMed: 481156].

43. Pandolf KB. Aging and human heat tolerance. Exp Aging Res. 1997;23(1):69-105. doi: 10.1080/03610739708254027. [PubMed: 9049613].

44. Ho CW, Beard JL, Farrell PA, Minson CT, Kenney WL. Age, fitness, and regional blood flow during exercise in the heat. J Appl Physiol (1985). 1997;82(4):1126-35. [PubMed: 9104849]. 
45. Food and Drug Administration. . Guidance for Magnetic Resonance Diagnostic Devices. Washington DC: Food and Drug Administration; 1997.
46. Oberschleissheim DE. Guidelines for limiting exposure to timevarying electric, magnetic and electromagnetic fields (up to 300 GHz). 1998. 